\title{
La reparación integral para las víctimas de violencia de género en el derecho constitucional
}

\section{Comprehensive reparation for victims of gender-based violence in constitutional law}

\author{
Germania Augusta Palomeque-Verdugo \\ palomequeg@psg.ucacue.edu.ec \\ Universidad Católica de Cuenca, Cuenca \\ Ecuador. \\ https://orcid.org/0000-0001-8239-7731 \\ Juan Carlos Erazo-Álvarez \\ jcerazo@ucacue.edu.ec \\ Universidad Católica de Cuenca, Cuenca \\ Ecuador \\ https://orcid.org/0000-0001-6480-2270 \\ Cecilia Ivonne Narváez-Zurita \\ inarvaez@ucacue.edu.ec \\ Universidad Católica de Cuenca, Cuenca \\ Ecuador \\ https://orcid.org/0000-0002-7437-9880 \\ José Luis Vázquez-Calle \\ jlvazquezc@ucacue.edu.ec \\ Universidad Católica de Cuenca, Cuenca \\ Ecuador \\ https://orcid.org/0000-0003-4980-6403
}

Recibido: 10 de abril de 2020

Revisado: 27 de abril de 2020

Aprobado: 26 de mayo de 2020

Publicado: 08 de junio de 2020

\section{RESUMEN}

La violencia de género ha sido uno de los problemas sociales en el Ecuador que no se ha podido superar y, si bien su constitución defiende la reparación integral, en la práctica no se cumple. El objetivo es determinar la efectividad de la reparación integral para las víctimas de violencia de género. La investigación fue no experimental, de tipo 
mixta con énfasis en lo cualitativo, los métodos utilizados fueron, inductivo-deductivo, histórico-lógico, analítico-sintético, y el derecho comparado. Los resultados mostraron que la reparación integral para las víctimas de violencia de género no es efectiva, debido a que se pudo observar que nadie la garantiza, quedando lejos todavía la reparación inmaterial sobre las víctimas en la que debería participar el Estado, estos resultados revelan que es necesario hacer cambios profundos, por ende, se creó una propuesta de un protocolo de acción para hacer un seguimiento y control.

Descriptores: Derechos de la mujer; derecho constitucional; derecho a la justicia; violencia. (Palabras tomadas del Tesauro UNESCO).

\section{ABSTRACT}

Gender violence has been one of the social problems in Ecuador that has not been overcome and, although its constitution defends comprehensive reparation, in practice it is not met. The objective is to determine the effectiveness of comprehensive reparation for victims of gender violence. The research was non-experimental, of a mixed type with an emphasis on the qualitative, the methods used were inductive-deductive, historicallogical, analytical-synthetic, and comparative law. The results showed that comprehensive reparation for victims of gender-based violence is not effective, since it was observed that no one guarantees it, and that immaterial reparation for victims in which the State should participate is still far away, these results reveal that It is necessary to make profound changes, therefore, a proposal for an action protocol was created to monitor and control.

Descriptors: Womens rights; constitutional law; right to justice; violence. (Words taken from the UNESCO Thesaurus).

\section{INTRODUCCIÓN}

El incremento de víctimas de violencia de género en el Ecuador ha producido una discusión constante sobre las formas en las que puede evitar este delito, así como sobre los medios para sancionar a los infractores y los elementos para resarcir el daño ocasionado a las personas que sufrieron este tipo de violencia. En los últimos años, se han creado varios cuerpos legales que han permitido que las personas que realizan este tipo de actos tengan que pagar las consecuencias de sus actos. Sin embargo, es necesario la reintegración de las víctimas a la sociedad de manera que el acto realizado tenga el menor impacto posible en su vida y las de su familia. 
Los mecanismos de reparación todavía siguen siendo incipientes e incompletos en el Ecuador, pese a la legislación que se ha venido construyendo mediante luchas de hace varias décadas. El papel desempeñado por los movimientos de mujeres y de género contra la violencia patriarcal y la lucha contra la discriminación tornaron que se visibilice la existencia de una problemática continua. En los años noventa los movimientos de género comenzaron a tener vigencia y ya con el nuevo milenio reclamaron constantemente reformas en las normativas y denunciaron la vulneración de sus derechos, así como también la violencia constante que debían soportar en el día a día.

La matriz discursiva de los derechos humanos fue clave cuando se retornó a la democracia en la región latinoamericana. Cobró protagonismo para los movimientos sociales ecuatorianos durante la Presidencia de León FebresCordero (1984-1988), mientras la violencia hacia las mujeres se posicionó como problema social en las calles. Este proceso estuvo acompañado de las conferencias y convenciones internacionales en las se definió la violencia hacia las mujeres como un problema de derechos humanos (ONU $1975,1979,1980,1993,1994)$. Tales iniciativas fueron un mecanismo para instar al Estado ecuatoriano a tomar medidas para prevenir, erradicar y sancionar este tipo de violencia. Varias ONGS dedicadas a trabajar por los derechos de las mujeres introdujeron enfáticamente la violencia de género en esta matriz discursiva en un escenario neoliberal (Guarderas-Albuja, 2016, p. 199).

La lucha contra la violencia de género no tiene un antecedente de pocos años, sino más bien representa las disputas de los movimientos sociales desde hace varias décadas. No obstante, el escenario actual de la legislación ecuatoriana ofrece una amplia gama de estructuras que ayudan en la construcción de mejores formas de erradicación y prevención de la violencia de género. Sin embargo, cuando la violencia acontecía, las maneras de reparar a la víctima se fundamentaban en llevar a prisión al victimario, multarlo, o hacer pagar una indemnización. En otras palabras, la reparación a la víctima era parcial, puesto que no existían mecanismos formales para ejecutar dicha reparación.

La reparación integral de las víctimas de violencia de género es un tema que no aparece de forma explícita en la Constitución de la República del Ecuador (CRE, 2008), pero se deriva de otros artículos en torno a la violencia y el derecho de las víctimas a la 
reparación. Si bien en la actualidad se cuenta con la Ley Orgánica para la Prevención y Erradicación de la Violencia de Género, la reparación integral puede tornarse engorrosa y muchas veces imposible de ser completada debido a la variedad de dimensiones que posee.

Como problema a solucionar se plantea ¿cómo mejorar la protección integral a las víctimas de violencia de género a partir de un estudio de casos de las sentencias del año 2019?, como objetivo proponer la aplicación de los estándares internacionales al momento de proteger y reparar integralmente a las víctimas de violencia de género para una mayor eficacia en el sistema. Esta propuesta servirá para colaborar con la justicia ecuatoriana en la constante lucha contra la impunidad y la educación de la sociedad en cuanto a considerar que reparar a las víctimas de la violencia de género no es solamente monetaria, sino tiene que ver con el empoderamiento, la protección a las mujeres, además de la educación, así como sanción a los victimarios, de tal manera que estos casos no se repitan.

\section{Referencial teórico}

\section{La reparación integral a partir de la Constitución de la República del Ecuador}

La responsabilidad civil tiene como marco el derecho privado y constituye un continuo objeto de discusión, pues como fundamento se encuentra el debate entre el derecho público y privado, que en la actualidad ya ha logrado ser superada, puesto que se considera al Derecho como único, por cuanto las normas aplicadas necesitan de un ente, en este caso el Estado, que aplique dichas normas, tanto para sanciones como para reparaciones de las víctimas.

Cuando existen daños de distinta índole, es responsabilidad del Estado velar para que se cumpla con la reparación de las víctimas, conforme lo establece la normativa. Ya no basta que la reparación sea solamente patrimonial sino también se deben considerar parámetros de otra índole que vayan encaminados a la reparación moral, ética e incluso no solamente de la víctima sino de la sociedad en general puesto que en la CRE y los diversos instrumentos legales internacionales se considera la protección a la dignidad 
del individuo y de los derechos humanos (Vásquez-Ayerve, Narváez-Zurita, PozoCabrera, \& Erazo-Álvarez, 2020).

El actual paradigma de responsabilidad civil establece la inviolabilidad del derecho de toda persona, de tal manera que, de ocurrir una transgresión de derechos, la reparación del daño esté más allá de lo meramente material. Precisamente, es en este sentido en el que se puede conceptualizar una reparación integral de casos de violencia de género. La idea es colocar en el núcleo de la violencia no sólo a la persona víctima, sino también a toda la familia, y es a esa persona y a la familia a la que se buscará reparar, pero esto significa trascender la visión material de la reparación ya que un individuo como tal es bastante complejo en sus dimensiones sociales, psicológica, y económicas (Ubiría, 2011).

La CRE en el artículo 78 determina que las víctimas recibirán una reparación que incluye "... el conocimiento de la verdad, restitución, rehabilitación, garantía de no repetición y satisfacción del derecho violado...". En el mismo orden de ideas, en el artículo 66 ibídem, se hace énfasis al derecho que tienen las personas a "... una vida libre de violencia en el ámbito público y privado" (CRE, 2008, art. 78). Del mismo modo, el artículo 81 ibídem establece que "la ley determinará los procedimientos especiales y expeditos para juzgar violencia intrafamiliar o sexual para persona vulnerables" (CRE, 2008, art. 81). Otro artículo que está relacionado es el artículo 331 que establece la prohibición de formas de violencia en contra de la mujer que le afecte en su trabajo (CRE, 2008, art. 331). El artículo 393 determina que el Estado garantizará la seguridad humana en cuanto a actos de violencia (CRE, 2008, art. 393, Asamblea Constituyente, 2008).

Todos estos artículos de la Constitución de la República muestran que el Estado tiene la responsabilidad de actuar en casos de violencia de género para establecer un proceso de reparación integral. El incremento de víctimas de violencia de género en el Ecuador, ha producido una discusión constante sobre las formas como puede evitarse, además de los medios para sancionar a los infractores, y los elementos para resarcir el daño ocasionado en las personas que sufrieron este tipo de violencia. En los últimos años, se han promulgado varios cuerpos legales que han permitido reprimir este tipo de infracciones con sanciones ejemplificadoras. 
Sin embargo, es necesaria la implementación de políticas de reparación que de manera efectiva reintegren a las víctimas a la sociedad de forma que el acto realizado tenga el menor impacto posible en su vida y las de su familia y además erradiquen este fenómeno social. Los mecanismos de reparación todavía siguen siendo incipientes e incompletos en el Ecuador, pese a que la legislación ha venido construyendo desde hace algunas décadas varias medidas de reparaciones tendientes a erradicar la violencia y a reducir el impacto físico, social y psicológico que provoca la violencia de género. El papel desempeñado por los movimientos de mujeres y su lucha por la igualdad de género contra la violencia patriarcal y la discriminación tornaron que se visibilice la existencia de una problemática continúa y enquistada profundamente en nuestra sociedad.

En los años noventa los movimientos de género comenzaron a tener vigencia, ya con el nuevo milenio reclamaron constantemente reformas en las normativas y denunciaron la vulneración de sus derechos, así como también la violencia constante que debían soportar en el día a día, fruto del sometimiento económico y de género que son víctimas (Ordóñez-Segarra, Narváez-Zurita, Vázquez-Calle, \& Erazo-Álvarez, 2020).

En Ecuador, la legislación sobre la reparación integral de víctimas de violencia de género está garantizada en la Constitución del año 2008 y tiene ya un instrumento diferenciado en la Ley de Prevención y Erradicación de la Violencia de Género, en el que se habla de manera amplia sobre los elementos que deben considerarse para el resarcimiento de las víctimas. No obstante, todavía existen situaciones que están demasiado vagas. Una de ellas tiene que ver con la subjetividad con la que el juez puede determinar la reparación, de acuerdo a su criterio, que puede terminar en injusticias, por lo que debería plantearse un esquema mucho más objetivo sistemático para el proceso (Machado, Medina, Vivanco, Goyas, \& Betancourt, 2018). 


\section{La problemática de la reparación integral a víctimas de violencia de género en el Ecuador}

Reconocer el derecho de las mujeres a una vida libre de violencia en la Constitución del Ecuador, tipificar el femicidio en el Código Orgánico Integral Penal (COIP), y la promulgación de la Ley de Erradicación y Prevención de la Violencia de Género son algunos de los pasos importantes que se han dado en el país en el aspecto legal para combatir la violencia de género. Sin embargo, debe mencionarse también que los procedimientos legales que siguen los jueces o las sentencias promulgadas no están de acuerdo con lo que se ha construido mediante estos cuerpos legales.

A tal punto sucede esto, que las cifras de violencia de género todavía tienen porcentajes altos. En el año 2014, en el Ecuador se publicó una encuesta referente a relaciones familiares y violencia de género contra las mujeres con datos obtenidos del año 2011 y se pudo observar que " 6 de cada 10 mujeres de 15 y más años $(60,6 \%)$ declaran haber vivido una o más formas de violencia de género, física, psicológica, sexual o patrimonial, por parte de cualquier persona conocida o extraña" (Camacho, 2014, p. 32), lo que denota claramente la existencia de un patrón que se mantiene pese a las medidas establecidas.

Las cifras muestran que los procedimientos legales y las decisiones de los jueces no han sido suficientes para contener el incremento de la violencia de género. Esta situación se hace mucho más grave cuando la reparación ejecutada se condiciona a niveles mínimos, es decir, que solamente se solicita aprehensión del agresor o una multa económica que no ayuda a solucionar la profundidad de las heridas causadas por el acto. Los jueces podrían llegar a minimizar algunos hechos de violencia, puesto que se han normalizado en la sociedad. Lo esencial es buscar ver la violencia como tal y no aceptarla (Gómez, 2014), tomando en cuenta que las causas de dicha violencia están arraigadas en las redes sociales, canciones, películas, videojuegos, propaganda, prensa, tradiciones, y más elementos culturales que la apoyan de manera sutil, lugares en los que la violencia es una estrategia de solución de problemas. En suma, la violencia no solamente se crea a partir de la relación de pareja, sino de una estructura 
social que provoca dicha violencia. Por ende, es necesario que la reparación incluya dentro de sus parámetros esta estructura social, haciendo responsable de la misma a la sociedad entera, de allí la denominación integral.

\section{Formas de Violencia}

Las Naciones Unidas ha realizado varios estudios para definir lo que se debe entender como violencia de género, partiendo de la situación de las mujeres en primera instancia, en general, se considera que "La violencia contra las mujeres no se confina a una cultura, región o país específico, ni a grupos particulares de mujeres en la sociedad. La violencia contra la mujer yace en la discriminación persistente contra las mujeres." (Naciones Unidas, 2009, p. 1). Extrapolando las expresiones en este artículo sobre el género, es posible establecer que la cultura de violencia está fundamentada en una estructura que la promulga y la normaliza.

Por otro lado, cabe señalar que la manera en la que se ve la violencia es reducida solamente al tipo físico, cuando en realidad se pueden presentar una variedad de violencia que va más allá de golpes, que inclusive podrían llegar a causar mucho más dolor que las formas comunes. A continuación, se describen algunos tipos en forma amplia:

a) Violencia contra la mujer en el ámbito de la pareja. - Es la forma de violencia más común que experimentan las mujeres en todo el mundo. Puede consistir en: a. Violencia física, que es el uso intencional de esta fuerza, pudiendo utilizar armas, con el objeto de dañar a la mujer. b. Violencia psicológica, las controla, aísla y humilla. La violencia económica les niega el acceso a los recursos básicos. c. Violencia sexual, que es la conducta por la cual se hace participar a la mujer en un acto sexual no consentido; realizarlo con una mujer incapacitada, o bajo los efectos de sustancias.

b) Violencia contra la mujer dentro de la familia. - La violencia que padecen muchas mujeres en el seno de la familia, puede comprender desde el período antes del nacimiento hasta la ancianidad. Se manifiesta en: a. Violencia física; violencia 
relacionada con la dote; b. Infanticidio femenino; abuso sexual de las niñas en el hogar; c. Ablación o mutilación genital; matrimonio precoz o forzado; d. Violencia ejercida por otros miembros de la familia. e. La violencia cometida contra las trabajadoras domésticas f. Otras formas de explotación. También, dentro de la familia, o al amparo de ésta, se cometen los denominados crímenes de "honor".

c) Violencia contra la mujer en la comunidad. - a. Feminicidio o femicidio: el homicidio de una mujer por cuestiones de género. b. Violencia sexual llevada a cabo fuera de la pareja. c. Acoso sexual y violencia en el lugar de trabajo, demás instituciones y en el ámbito deportivo. Un 50\% aproximadamente de mujeres de la Unión Europea manifiestan haber sido acosadas en alguna ocasión en sus puestos de trabajo. d. Trata de mujeres: la mayoría de las víctimas de trata de seres humanos en el mundo son mujeres y niños. e. Otras violencias: dedicación de las niñas pequeñas a templos. Las restricciones de la segunda hija a casarse. El matrimonio con el hermano del marido fallecido. El maltrato de las viudas, en particular la incitación a que se suiciden.

d) Violencia contra la mujer cometida o tolerada por el Estado. - a. Violencia contra la mujer privada de libertad: es la que se lleva a cabo en prisiones, centros de bienestar social, etc. b. Esterilización forzada: controla la reproducción de la población femenina, o de un subgrupo determinado.

e) Violencia contra la mujer en conflictos armados.- Como consecuencia de las guerras, las mujeres padecen todo tipo de violencia física, sexual y psicológica, comprendiendo: a. Homicidios, torturas, raptos, mutilaciones y desfiguraciones, reclutamiento forzado de mujeres combatientes; b. Violaciones, esclavitud sexual, explotación sexual, prostitución forzada; c. Desapariciones involuntarias, prisiones arbitrarias, matrimonios forzados; d. Abortos forzados, embarazos forzados; esterilización compulsiva. (Yungueros-García, 2014, p. 151-152)

Como puede verse, la violencia de género no está restringida a un solo tipo, por ende, tampoco puede entender la misma reparación para todos los casos que se analicen. Estas formas de violencia tienen como fundamento esencial la subordinación a un 
patriarcado que se ha ido fomentando desde el orden social, cultural, económico. Esta idea ha ido elaborándose conjuntamente con la categoría de género que es necesario entender para configurar aquello que se ha plasmado en las leyes del país, en la consiguiente reparación que debe hacerse cuando se ejecutan hechos de violencia.

El género busca explicar de manera objetiva las relaciones entre hombres y mujeres, de acuerdo al significado que les han otorgado a sus vidas, a partir de su sexualidad y los dominios del poder (Peñafiel-Bermeo, Narváez-Zurita, Vázquez-Calle, \& ErazoÁlvarez, 2020). En general, se critica la designación del elemento biológico como la manera en la que se designan las identidades y los roles. En consecuencia, el género se define en dos aspectos:

Es un elemento constitutivo de las relaciones sociales basadas en las diferencias que distinguen los sexos y el género es una forma primaria de relaciones significantes de poder. Conceptos normativos que manifiestan las interpretaciones de los significados de los símbolos, en un intento de limitar y contener sus posibilidades metafóricas. Este enfoque permite establecer que la violencia de género no surge de una espontánea disputa entre un hombre y una mujer, sino que está subordinada a una estructura social latente en la que el hombre prevalece dentro del ámbito privado, por lo que se determina que cualquier tipo de sublevación a su poder intrínseco y otorgado por la sociedad y la cultura, debe ser exterminado a través de la violencia.

Mientras tanto, en el entorno público la violencia de género tiene otras formas, pero tiene una estructura similar, manifestándose principalmente en conflictos armados, violencia estatal, entre otras, como se vio anteriormente en los tipos de violencia. Cabe mencionar, que, si bien en este trabajo se ha analizado la violencia de género por ser un concepto más amplio, son varios los instrumentos que se han trabajado durante décadas para erradicar la violencia contra la mujer. Para la Convención Interamericana para Prevenir, Sancionar y Erradicar la Violencia contra la Mujer, comúnmente conocida como Belém do Pará, promulgada en el año de 1994, se considera como violencia contra la mujer lo siguiente: 
a) Que tenga lugar dentro de la familia o unidad doméstica o en cualquier otra relación interpersonal, ya sea que el agresor comparta o haya compartido el mismo domicilio que la mujer, y que comprende, entre otros, violación, maltrato y abuso sexual;

b) Que tenga lugar en la comunidad y sea perpetrada por cualquier persona y que comprende, entre otros, violación, abuso sexual, tortura, trata de personas, prostitución forzada, secuestro y acoso sexual en el lugar de trabajo, así como en instituciones educativas, establecimientos de salud o cualquier otro lugar, y

c) Que sea perpetrada o tolerada por el Estado o sus agentes, donde quiera que ocurra. (Departamento de Derecho Internacional OEA, 1994)

En consecuencia, la violencia de género es una manifestación de la desigualdad de poder entre los géneros que se encuentran en disputa, acentuada por las estructuras socioculturales instauradas en la sociedad. La vulnerabilidad de las mujeres y de otros géneros a la violencia está condicionada por diversos factores que deben tomarse en cuenta al momento de plantear una efectiva reparación integral de la violencia. La Organización Mundial de la Salud (OMS, 2017) plantea como factores de vulnerabilidad a la violencia los siguientes:

a) Entre los factores generales se describen los siguientes:

- Bajo nivel de instrucción de los autores de violencia sexual y víctimas de violencia sexual.

- La exposición al maltrato infantil de autores y víctimas.

- La experiencia de violencia familiar de autores y víctimas.

- El trastorno de personalidad antisocial de los autores.

- El uso nocivo del alcohol de autores y de las víctimas.

- Tener muchas parejas o de inspirar sospechas de infidelidad en la pareja (autores).

- Las actitudes de aceptación de la violencia (autores y víctimas).

b) Entre los factores asociados específicamente a la violencia de pareja; se señalan: 
- Los antecedentes de violencia (autores y víctimas);

- La discordia e insatisfacción marital (autores y víctimas).

- Las dificultades de comunicación entre los miembros de la pareja.

c) Los factores asociados específicamente a la violencia sexual; se mencionan:

- La creencia en el honor de la familia y la pureza sexual.

- Las ideologías que consagran los privilegios sexuales del hombre.

- La levedad de las sanciones legales contra los actos de violencia sexual. (Organización Mundial de la Salud, 2017, p. 2)

En el Ecuador existen algunas sentencias que pueden ser de importancia para entender cómo se ha venido dando la reparación de las víctimas de violencia de género. Por ejemplo, en una investigación realizada por diario El Telégrafo se pudo establecer que en el $99 \%$ de los casos de femicidios, no se procede a la reparación integral, inclusive no se llega a realizar el resarcimiento económico, puesto que una cantidad de casos grandes se dan en hogares de escasos recursos económicos (El Telégrafo, 2019). Dentro de las sentencias puede verse además claramente una perspectiva económica por parte de los jueces.

En el año 2015 dentro del juicio Nro. 01283-2015-06473 en la ciudad de Cuenca, se consideró una reparación de 500 dólares luego de que el victimario agredió a palos a su entonces novia; en la sentencia no se establece reparación psicológica o social. Otro caso similar, de acuerdo al juicio Nro. 01283-2015-06503, ocurrió cuando un esposo roció a su víctima con una sustancia desconocida provocándole quemaduras en su piel, que le dejaron inhabilitada por más de un mes; en este caso la sentencia condena a prisión al victimario, se establece una reparación económica, aunque aquí sí se reconoce la garantía de no repetición y derecho al conocimiento de la verdad.

No obstante, en este caso, tampoco se establece una reparación integra al no solicitar terapia psicológica para la afectada. Este tipo de casos, son de sentencias anteriores a la Ley Orgánica para Prevenir y Erradicar la Violencia de Género, por lo que cabría hacer un análisis de lo que sucede ahora para proceder justamente a la realización de una propuesta diferente (Martínez-Leguízamo, 2018). 
En suma, la vulnerabilidad expone a las mujeres y otros géneros a recibir todo tipo de violencia, por lo que cuando se piensa en reparación integral es necesario ser conscientes que estas personas no pueden volver a situaciones de vulnerabilidad puesto que posiblemente la violencia volverá a suceder. Bajo estos conceptos, se han elaborado los cuerpos legales que funcionan actualmente en el Ecuador y que se analizan a continuación a nivel nacional e internacional.

\section{Estándares internacionales aplicables a la reparación integral a víctimas de violencia de género en el Ecuador}

Los estudios sobre la relación de violencia de género y la aplicación de los cuerpos legales se ha extendido en los últimos años considerándose el análisis de los factores con los que se estructuraron los diferentes cuerpos legales (Quiñonez, Zambrano \& Prado, 2017), y uno de los temas es la reparación integral de la violencia de género. Esta nunca puede ser reducida a la violencia sexual, y tampoco puede ser confundido con el feminicidio. Las dimensiones de la violencia de género van más allá de la violencia sexual, y tiene que ver con violencia simbólica, estructural y económica (Benavides-Vanegas, 2015), por lo que la reparación integral debe también tener aquellos criterios, por ende, las dimensiones de la reparación integral son también más amplias.

Los cuerpos legales creados en los últimos años, como la Constitución de la República del Ecuador, 2008 y la Ley de Prevención y Erradicación de la Violencia de Género, han traído seguridad jurídica para la reparación integral, pero la judicialización del ámbito privado donde se presenta la violencia muchas veces expone a la víctima a mayor grado de sufrimiento (Guarderas-Albuja, 2016), más aun, cuando la reparación no se ejecuta de forma integral. Luchar contra la impunidad y tratar de reparar a las víctimas de la violencia de género, no es solamente dar dinero a las víctimas, sino empoderar y proteger a las mujeres, además de educar y sancionar a los victimarios para que estos casos no se repitan (Saccomano, 2017). A continuación, se presentan algunas características a nivel internacional, regional y nacional. 
En el contexto internacional, los tribunales penales internacionales en casos como los de Ruanda y la ex Yugoslavia, se crearon órdenes de restitución que estaban basados en la parte netamente económica. La Corte Penal Internacional ha avanzado en la materia de restitución hasta llegar al punto en el que las víctimas pueden ser reparadas más, sin embargo, esta reparación muchas veces no llega a ser integral. La Corte Interamericana de Derechos Humanos amplía dicha visión y habla de "otras formas de reparación", aunque en la jurisprudencia revisada, la complejidad de los casos hace dificultoso que se apliquen de manera efectiva reparaciones integrales, y aunque se establezcan las medidas, otra cosa es la aplicación por parte del Estado, puesto que muchas veces el cumplimiento toma varios años, llegando a la re-victimización (AriasLópez, 2017).

A nivel regional, la reparación integral de las víctimas de violencia de género ha tenido mayor acogida, especialmente en aquellos países en donde han existido conflictos militares a gran escala. Por ejemplo, en Colombia se ha generado una discusión sobre la manera en la que se debe reparar de forma integral a las víctimas de violencia de género desde un enfoque diferencial y tomando en cuenta la múltiple victimización, pues son víctimas del conflicto armado y de la violencia de género.

La integralidad en este contexto, es reparar las víctimas en todas sus esferas al momento en que se ejecuten los hechos que vulneraron sus derechos humanos (Sánchez \& Olivares, 2014). La reparación de los daños de acuerdo a este enfoque establece que la mujer debe superar los daños que se hacen exclusivamente a su integridad sexual (González-Cárdenas, Narváez-Zurita, Guerra-Coronel, \& ErazoÁlvarez, 2020).

El Estado colombiano ha materializado el enfoque de reparación a través de programas de atención, y en muchos de los casos estos programas han transformado estructuralmente la situación de las víctimas. El conflicto armado colombiano ha mejorado la visión sobre la reparación integral de las mujeres víctimas de la guerra y la violencia de género, volviéndola más amplia (Castrellón-Pérez \& Romero-Cristancho, 2016). También se ha planteado que la reparación puede dividirse en medidas de 
efecto directo, efecto general, efecto vinculante, y por su naturaleza (Restitución, Indemnización, Rehabilitación, No Repetición, y Satisfacción).

Esta división de las formas de reparación tiene como resultado una mejor comprensión de la integralidad de la reparación. El propósito es dignificar y restituir a las víctimas en su derecho, y que las violaciones cometidas no vuelvan a realizarse nunca más, en lo cual el Estado tiene un papel fundamental, para lograr el mayor grado de restitución posible, puesto que una víctima muy posiblemente nunca sea reparada en su totalidad (Chinchilla-Fuentes, 2018).

También es menester considerar los esfuerzos que han hecho algunas entidades públicas al respecto, como el documento del Distrito Metropolitano de Quito denominado "Ruta de atención, protección y restitución de derechos de mujeres en situación de violencia en el marco del sistema de protección integral", elaborado en el 2017, en el que se plantean formas de asistencia, protección, restitución, y reparación integral en casos de violencia en contra de las mujeres, estableciendo que son varios los organismos que deben entrar en la reparación y que la detección de incumplimiento establece precisamente una vulneración de derechos (CPD, Red RAP VIF SUR, 2017). No obstante, la reparación integral a las víctimas tampoco es un tema nuevo en el Ecuador, puesto que, a raíz de los crímenes de lesa humanidad efectuado en las décadas de los setentas y ochentas, se crearon programas con estos objetivos como el denominado "Programa Nacional de Reparación a Víctimas" que estaba a cargo de la Defensoría del Pueblo que se encargaba de la reparación inmaterial, mientras que el Ministerio de Justicia, Derechos Humanos y Cultos ejecutaba la reparación material. Los problemas de estas formas de reparación estuvieron en las formalidades y los procedimientos, olvidando el lado humano de las personas víctimas de violencia, y este enfoque de reparación fue el que se mantuvo por varios años, dejando a un lado precisamente la integralidad (Solís, 2018). La acción de protección respaldada por la Constitución fue otra manera de reparar a la víctima, pero tenía problemas pues dependía del juzgador constitucional (López-Zambrano, 2018). 
Por este motivo, en el año 2017 se presenta en la Asamblea Nacional la Ley Orgánica Integral para la Prevención y Erradicación de la Violencia de Género donde se planteó la reparación integral de las víctimas contemplando cinco tipos de violencia (física, psicológica, sexual, patrimonial y simbólica), con sus respectivos ámbitos (político, educativo, institucional, judicial, familiar, y comunitario) (Berni, 2018).

La elaboración de esta ley es muy importante puesto que incorpora un enfoque que no estaba determinado y busca particularizar los programas de prevención y la manera en la que se brinda asistencia a las víctimas de violencia de género. Son varios los cuestionamientos que se han generado, sin embargo, la Corte Nacional de Justicia resolvió que la Ley tenía concordancia con los cuerpos legales vigentes, por lo que los jueces tienen seguridad jurídica para sus actuaciones (Guzmán, Vaca, Goyas \& Machado, 2019). La reparación integral de la violencia de género debe incluir un análisis de los factores económicos, políticos, y de salud.

La incidencia que tienen los títulos de propiedad de tierras y viviendas, la alfabetización judicial de las mujeres, el empoderamiento económico, la educación de las víctimas, entre otras (Vélez-Valencia, 2017). Asimismo, existen otros temas tales como el acompañamiento psicosocial y psiquiátrico, indemnizaciones económicas, el reconocimiento de responsabilidad por parte del Estado, acercamiento de la justicia hacia las víctimas, la institucionalización de los procesos para la reparación integral, la elaboración de programas, entre otros (Salcedo-López, 2013). La CIDH recomienda también medidas de reparación conjuntamente con aquellas de prevención y encamina a los Estados a crear y aplicar cuerpos legales y políticas con la intención de crear un ambiente de no violencia (Taus, 2014).

\section{MÉTODO}

La metodología se fundamentó en un diseño no experimental puesto que se realizó un estudio de las variables tal como aparecen sin ser manipuladas. Además, se planteó un estudio mixto (cuantitativo-cualitativo), con mayor énfasis en el análisis cualitativo de la información documental-bibliográfica, aunque también se buscó tener datos 
cuantitativos mediante la aplicación de una encuesta (Hernández \& Mendoza, 2018).

Se estableció como métodos generales el Inductivo-deductivo para determinar desde datos generales una conclusión, además del Histórico-Lógico al considerar el contexto en la secuencia cronológica, el comparativo que permite tener herramientas jurídicas propias de la investigación que se está llevando a cabo (Hernández, Fernández, \& Baptista, 2014).

Los métodos utilizados permitieron el análisis de la doctrina, las leyes, las sentencias, entre otros documentos como fundamento para el estudio del problema en el contexto del Derecho Constitucional. Se ha aplicado además la técnica del estudio de caso para revisar sentencias jurídicas específicas que aclaran la aplicación de la reparación integral en casos de violencia de género (Peñafiel-Sacoto, Erazo-Álvarez, PozoCabrera, \& Narváez-Zurita, 2020). Se aplicó también una encuesta a servidores judiciales para establecer su percepción sobre la reparación integral en el sistema judicial.

\section{Universo de estudio y tratamiento muestral}

Se ha utilizado el muestreo por conveniencia, técnica de muestreo no probabilístico y aleatorio en el que se toma a casos que se encuentran disponibles a los que se tiene acceso para que formen parte del estudio, para lo que se ha establecido una muestra de 20 operadores de la función judicial.

\section{Tratamiento estadístico de la información}

Los datos se obtuvieron a través de cuestionarios y formularios enviados mediante Google Forms, que a su vez se procesaron en tablas de datos con las respuestas de las personas, tabuladas mediante el programa Microsoft Excel 2019, cuya información sirvió para el análisis respectivo. 


\section{RESULTADOS}

A continuación, se presenta la tabla y la figura 1 con el extracto de los resultados adquiridos, clasificados según las variables definidas en el instrumento de investigación aplicado:

\section{Tabla 1}

Resultados de la encuesta aplicada a funcionarios de la función judicial

\begin{tabular}{|c|c|c|c|}
\hline \multirow[b]{2}{*}{ Variable } & \multirow[b]{2}{*}{ Pregunta } & \multicolumn{2}{|c|}{ Resultado \% } \\
\hline & & Si & No \\
\hline \multirow[t]{4}{*}{$\begin{array}{l}\text { Violencia de } \\
\text { género }\end{array}$} & $\begin{array}{l}\text { 1. ¿Cree usted que se pueda prevenir y erradicar la } \\
\text { violencia de género en nuestro país? }\end{array}$ & $60.00 \%$ & $40.00 \%$ \\
\hline & $\begin{array}{l}\text { 2. ¿Cree usted que en los últimos años la violencia de } \\
\text { género en nuestro país se ha incrementado? }\end{array}$ & $65.00 \%$ & $35.00 \%$ \\
\hline & $\begin{array}{l}\text { 3. ¿Cree usted que en nuestro país se están tomando } \\
\text { medidas para poner fin a la violencia de género? }\end{array}$ & $80.00 \%$ & $20.00 \%$ \\
\hline & $\begin{array}{l}\text { 5. ¿Cree usted que la reparación integral para las víctimas } \\
\text { de violencia de género en nuestro país es efectiva? }\end{array}$ & $15.00 \%$ & $85.00 \%$ \\
\hline \multirow{3}{*}{$\begin{array}{l}\text { Reparación } \\
\text { Integral }\end{array}$} & $\begin{array}{l}\text { 6. ¿Cree usted que la reparación integral para las víctimas } \\
\text { de violencia de género debe incluir una faceta individual y } \\
\text { colectiva? }\end{array}$ & $100.00 \%$ & $0.00 \%$ \\
\hline & $\begin{array}{l}\text { 7. ¿Cree usted que la reparación integral para las víctimas } \\
\text { de violencia de género debe ser material e inmaterial? }\end{array}$ & $95.00 \%$ & $5.00 \%$ \\
\hline & $\begin{array}{l}\text { 8. ¿Cree usted que la reparación integral se cumple tal y } \\
\text { como se menciona en las sentencias? }\end{array}$ & $10.00 \%$ & $90.00 \%$ \\
\hline
\end{tabular}

Fuente: Investigación de campo 


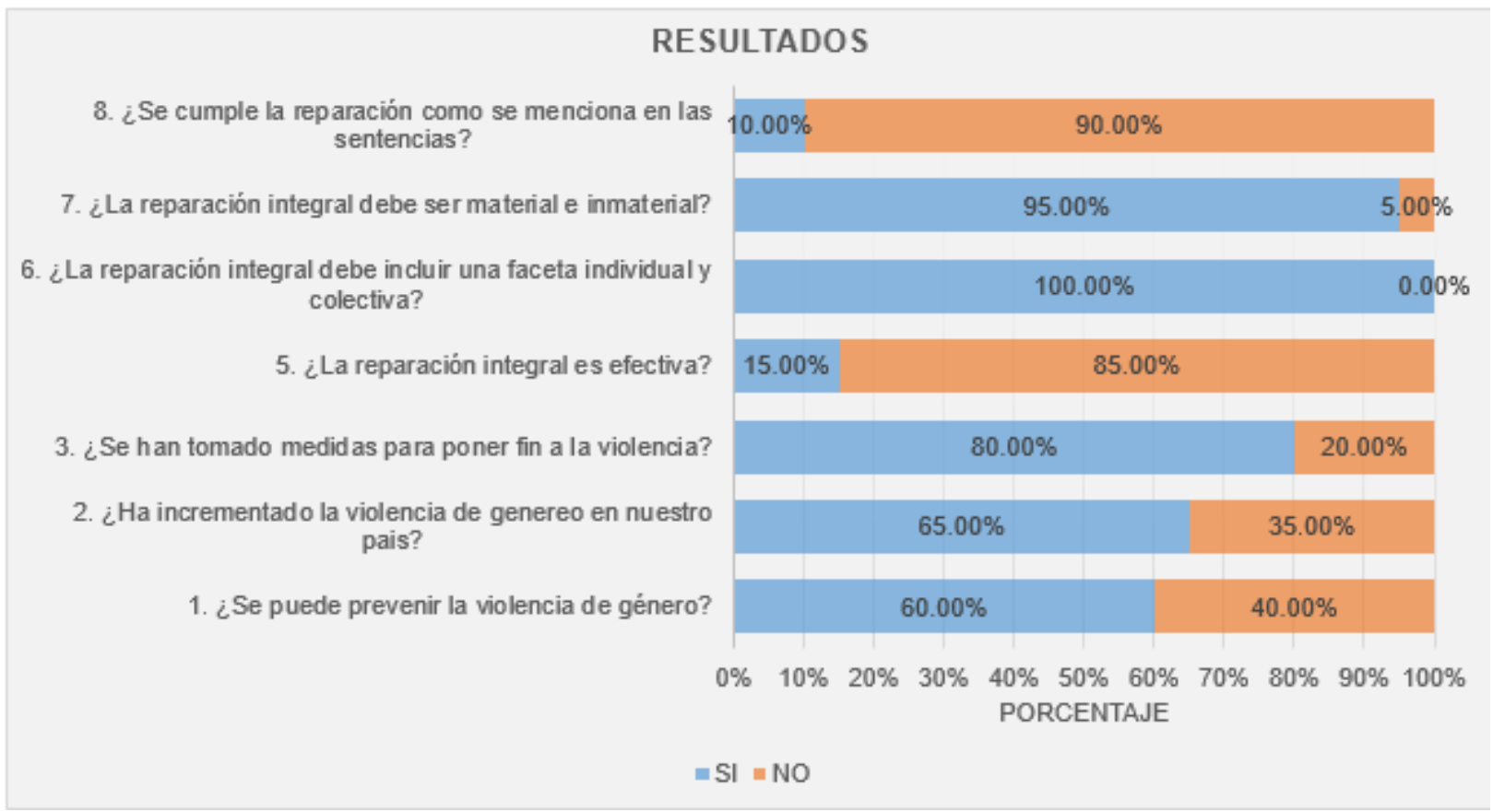

Figura 1 Representación gráfica de los resultados. Fuente: Investigación de campo Se consultaron a funcionarios judiciales sobre la violencia de género y la reparación integral de las víctimas. Las interrogantes se realizaron con el objetivo de realizar un análisis jurídico y entender posibles tendencias de género que influencien en las opiniones. Las respuestas mostraron que la reparación integral no es efectiva, así como que no se cumple en su totalidad.

\section{Violencia de género}

Sobre este punto y conforme se demuestra en la tabla y figura 1, se entendió que sí es posible erradicar y prevenir la violencia de género. Otro elemento importante demostró que la creencia de los funcionarios judiciales encuestados (que son quienes receptan las denuncias sobre este caso) es que la violencia de género en el país ha incrementado, lo que se corrobora con la información que indica que en el $64 \%$ de los hogares se mantiene en su núcleo casos de violencia intrafamiliar. Se entendió también que el Ecuador está adoptando medidas necesarias para combatir y poner fin a la violencia de género producto de la aprobación de la Ley para Prevenir y Erradicar la 
Violencia de Género contra las Mujeres.

\section{Reparación Integral}

Se demostró que reparación integral en el Ecuador no es efectiva; al emitirse una sentencia relacionada con la violencia de género no diseña adecuadamente la reparación. Además, esta reparación debe constar de una faceta individual y colectiva, que incluya a familiares de la víctima, medios de comunicación y elementos económicos que garanticen la reintegración de la persona a la sociedad, de igual forma debe ser material e inmaterial incluyendo tratamiento psicológico para la víctima, los familiares y el victimario, además de la recuperación del honor de la víctima. Por último, se expuso que la reparación no se cumple tal y como se dispone en las sentencias, ya que la reparación económica muchas veces no llega a darse.

Se puede concluir señalando que, a pesar de los esfuerzos por prevenir y erradicar la violencia de género a nivel nacional, al momento de juzgarse no se realiza de forma integral la reparación, desnaturalizándose su concepción, lo que a su vez demuestra que existen debilidades del sistema judicial ecuatoriano en cuanto los derechos de las víctimas de violencia de género.

\section{PROPUESTA}

La propuesta de la investigación realizada implica la expedición de un protocolo de seguimiento y cumplimiento de la reparación integral de las víctimas de violencia de género, la sociabilización, análisis y discusión de los artículos para mejorar el protocolo que dará paso a la aplicación de esta herramienta con la finalidad de fortalecer las sentencias sobre violencia de género y el esfuerzo de los operadores de justicia para que la víctima quede satisfecha con lo actuado por la función judicial. 

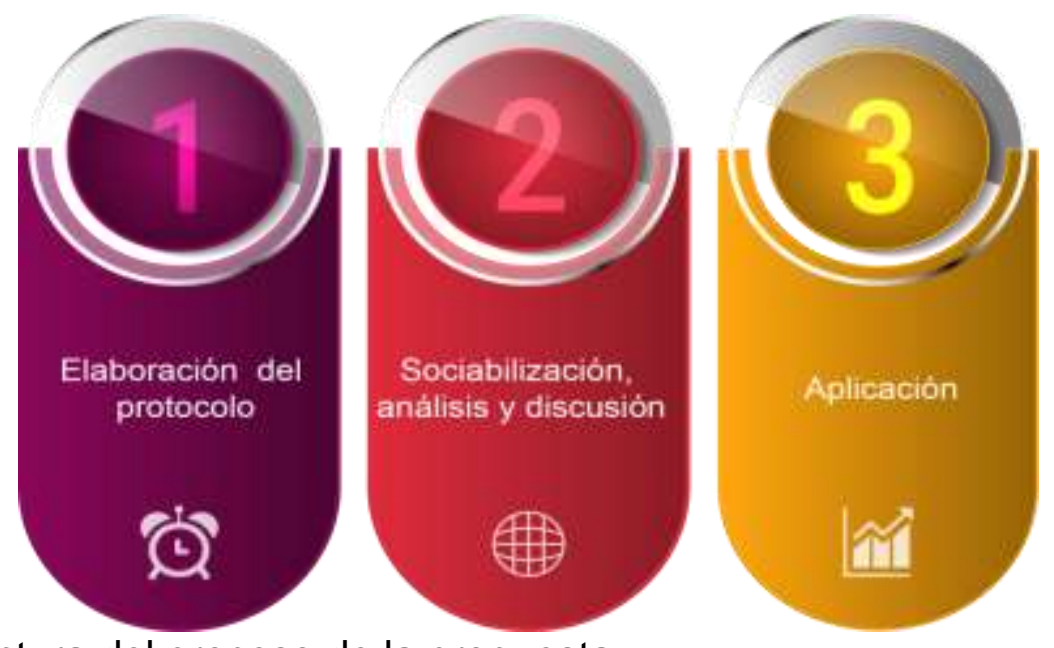

Figura 2. Estructura del proceso de la propuesta

\section{RESOLUCIÓN 054-2020 \\ EL PLENO DEL CONSEJO DE LA JUDICATURA CONSIDERADO:}

Que el artículo 168 numeral 2 de la Constitución de la República del Ecuador, determina: "La administración de justicia, en el cumplimiento de sus deberes y en el ejercicio de sus atribuciones, aplicará los siguientes principios: 2. La Función Judicial gozará de autonomía administrativa, económica y financiera.";

Que el artículo 177 de la Constitución de la República del Ecuador, establece: "La Función Judicial se compone de órganos jurisdiccionales, órganos administrativos, órganos auxiliares y órganos autónomos. La ley determinará su estructura, funciones, atribuciones, competencias y todo lo necesario para la adecuada administración de justicia";

Que el artículo 178 inciso segundo de la Constitución de la República del Ecuador, así como el artículo 254 del Código Orgánico de la Función Judicial, prevén que el Consejo de la Judicatura es el órgano de gobierno, administración, vigilancia y disciplina de la Función Judicial, 
Que el artículo 100 numeral 1 del Código Orgánico de la Función Judicial, manifiestan: "DEBERES.- Son deberes de las servidoras y servidores de la Función Judicial, según corresponda al puesto que desempeñen, los siguientes: 1. Cumplir, hacer cumplir y aplicar, dentro del ámbito de sus funciones, la Constitución, los instrumentos internacionales de derechos humanos, las leyes y reglamentos generales; el Estatuto Orgánico Administrativo de la Función Judicial, los reglamentos, manuales, instructivos y resoluciones del Pleno del Consejo de la Judicatura y de sus superiores jerárquicos;

Que el artículo 78 de la Constitución de la República del Ecuador dispone: "Las víctimas de infracciones penales gozarán de protección especial, se les garantizará su no revictimización, particularmente en la obtención y valoración de las pruebas, y se las protegerá de cualquier amenaza u otras formas de intimidación.

Que el artículo 81 de la Constitución de la República del Ecuador señala: "La ley establecerá procedimientos especiales y expeditos para el juzgamiento y sanción de los delitos de violencia intrafamiliar, sexual, crímenes de odio y los que se cometan contra niñas, niños, adolescentes, jóvenes, personas con discapacidad, adultas mayores y personas que, por sus particularidades, requieren una mayor protección. Se nombrarán fiscales y defensoras o defensores especializados para el tratamiento de estas causas, de acuerdo con la ley".

Que el artículo 331 de la Constitución de la República del Ecuador dispone: "El Estado garantizará a las mujeres igualdad en el acceso al empleo, a la formación y promoción laboral y profesional, a la remuneración equitativa, y a la iniciativa de trabajo autónomo. Se adoptarán todas las medidas necesarias para eliminar las desigualdades. Se prohíbe toda forma de discriminación, acoso o acto de violencia de cualquier índole, sea directa o indirecta, que afecte a las mujeres en el trabajo".

Que el artículo 393 de la Constitución de la República del Ecuador dispone: "El Estado garantizará la seguridad humana a través de políticas y acciones integradas, para asegurar la convivencia pacífica de las personas, promover una cultura de paz y prevenir las formas de violencia y discriminación y la comisión de infracciones y delitos". 
La planificación y aplicación de estas políticas se encargará a órganos especializados en los diferentes niveles de gobierno.

En ejercicio de sus atribuciones constitucionales y legales contenidas en los artículos 181 de la Constitución de la República del Ecuador y 264 numerales 1 y 10 del Código Orgánico de la Función Judicial,

\section{RESUELVE:}

\section{EXPEDIR EL PROTOCOLO DE SEGUIMIENTO Y CUMPLIMIENTO DE LA REPARACIÓN INTEGRAL DE LAS VÍCTIMAS DE VIOLENCIA DE GÉNERO. \\ Artículo 1.- Objeto}

El presente protocolo, tiene como esencia dar seguimiento y cumplimiento a la reparación integral de las víctimas de violencia de género, generada en el entorno público y privado.

\section{Artículo 2.- Ámbito de aplicación}

El presente protocolo será aplicable a toda persona natural o jurídica, que se encuentre o actúe en el territorio ecuatoriano.

\section{Articulo 3.- Fases de trabajo:}

1) Fase de capacitación. - En la primera fase se pretende capacitar a toda la función judicial sobre el trato de casos de violencia de género, para que se entienda claramente el papel del sistema de justicia y la importancia de realizar el seguimiento a la reparación integral. Las conclusiones de la capacitación servirán para la construcción del protocolo.

2) Fase de mesas de trabajo. - La segunda fase, se realizará con la participación de diferentes grupos de la sociedad, entre ellos, grupos defensores de los derechos de género, víctimas de la violencia de género, y otros. En las mesas de trabajo se establecerán los puntos medulares sobre las actuales falencias del sistema de reparación integral de la actualidad en cuanto a la aplicación de la misma.

3) Veedurías ciudadanas. - Debe conformarse una veeduría ciudadana conformada por organizaciones sociales que puedan denunciar cuando la justicia no logra 
aplicar con rigurosidad la reparación integral.

\section{Articulo 4.- Reparación material}

Si las sentencias establecieron que el individuo debe pagar una suma de dinero por el daño realizado, esto debería cumplirse, aunque el acusado no tenga los medios económicos para pagar. En este caso, es el Estado el que debería asumir los pagos si es que la víctima no recibe la compensación económica de la sentencia.

\section{Articulo 5.- Reparación inmaterial}

El Estado debe poner a disposición de las víctimas todos los medios que tenga a disposición para que las víctimas y sus familiares reciban atención psicológica, la garantía a la no repetición, además de ser reintegrados a la sociedad tanto en su trabajo como en su comunidad, buscando que no se les estigmatice de ninguna manera.

\section{Articulo 6.- Oficina especial}

La función judicial debe crear una oficina especializada en el seguimiento y difusión de los casos de violencia de género y su reparación integral, de tal manera que las personas conozcan que la justicia ha logrado su objetivo.

\section{Articulo 7.- Rehabilitación de victimarios}

La reparación será integral también si es que el victimario es sometido a diversas sesiones de terapia psicológica y trabajo comunitario en el que pueda palpar la realidad de sus acciones.

Notifíquese, publíquese en la página web y cúmplase.

Dada en el Distrito Metropolitano de Quito, a los .... días del mes de mayo de dos mil veinte.

\section{CONCLUSIONES}

La violencia de género en el Ecuador cada día tiene un mayor número de víctimas, además de no tener protocolos claros de actuación por parte de los operadores de justicia para ejecutar las sentencias, de tal manera que se consiga la reparación integral consagrada en la Constitución y la protección integral de las víctimas de estos delitos. 
De acuerdo con el objetivo de este artículo, se pudo determinar las falencias que tiene el cumplimiento de la reparación integral en las víctimas de violencia de género, a través de las opiniones de expertos en el área del Derecho y del análisis de las leyes vigentes, sentencias ejecutoriadas, y teoría doctrinaria. Entre las principales debilidades está la excesiva atención que se brinda a lo punitivo de las sentencias, es decir, buscar que el victimario vaya a la cárcel o pague la multa económica, más, sin embargo, lo esencial, que sería tratar de reparar el daño moral, psicológico y social de la víctima, no se toma con la misma responsabilidad, ni tampoco se la brinda el seguimiento adecuado.

Además, se pudo determinar que algunas sentencias simplemente terminan en la reparación material, dejando a un lado la reparación inmaterial, determinando multas que muchas veces no son pagadas por la escasez de recursos económicos del victimario. Otro de los temas importantes analizados a través de la encuesta realizada a operadores de justicia que trabajan en el área, fue la poca o nula capacidad del sistema de justicia para hacer el correcto seguimiento y control del cumplimiento de la reparación integral, debido en parte al tiempo y la dificultad que resulta determinar terapia psicológica para la víctima y la familia por varios meses o incluso años, usar medios de comunicación para que la víctima pueda hablar sobre lo ocurrido, e inclusive otros temas como la reintegración a la sociedad.

Por ende, se determinó como indispensable que se cumpla con la norma constitucional y con las leyes de protección a víctimas de violencia de género, mediante la creación de un protocolo de seguimiento y control de la reparación integral que esté compuesta de varios elementos tales como la creación de veedurías ciudadanas, capacitación a los operadores de justicia y trabajo constante con víctimas, sus familias y los victimarios. La discusión doctrinaria y de aplicación de justicia todavía tiene muchas aristas en donde seguir ahondando sobre la calidad de las sentencias y su conflicto con las normativas constitucionales que muchas veces no son cumplidas por falta de una ley, un reglamento o un protocolo de actuación. En consecuencia, trabajos como este tienen la labor de contribuir para reparar y corregir actuaciones inconstitucionales como el de 
la reparación integral en el caso de violencia de género en el Ecuador.

\section{FINANCIAMIENTO}

No monetario.

\section{AGRADECIMIENTO}

A la Universidad Católica de Cuenca, por incentivar el desarrollo de la investigación.

\section{REFERENCIAS CONSULTADAS}

Arias-López, B. (2017). La reparación integral en el proceso penal Boliviano. [Integral reparation in the Bolivian penal process]. Revista Jurídica Derecho, 5(6), 49-66. Recuperado de https://cutt.ly/myliDyX

Asamblea Constituyente (2008). Constitución de la Rep[ublica del Ecuador 2008. [Constitution of the Republic of Ecuador 2008]. Derechos de protección. Montecristi, Ecuador: Registro Oficial No. 449.

Benavides-Vanegas, F. (2015) Feminicidio y derecho penal. Feminicide and criminal law]. Revista Criminalidad, 57 (1), 75-90. Recuperado de https://cutt.ly/MylorbF

Berni, M. (2018). Estrativismo social machista en Ecuador violencia de género, femicidio. [Machist social estrativism in Ecuador gender violence, femicide]. Conrado, 14(61), 111-115. Recuperado de https://cutt.ly/NylopX2

Camacho, G. (2014). La violencia de género contra las mujeres en el Ecuador: Análisis de los resultados de la Encuesta Nacional sobre Relaciones Familiares y Violencia de Género contra las Mujeres. [gender-based violence against women in Ecuador: Analysis of the results of the National Survey on Family Relations and Gender Violence against Women]. Recuperado de https://url2.cl/ulma6

Castrellón-Pérez, M, \& Romero-Cristancho, C. (2016). Enfoque de género en la implementación de la Ley de Víctimas y Restitución de Tierras: una propuesta para la caracterización de las mujeres y niñas víctimas del conflicto armado en Colombia. [Gender approach in the implementation of the Victims and Land Restitution Law: a proposal for the characterization of women and girls victims of the armed conflict in Colombia]. Revista CS, no. 19, pp 69-113. 
Chinchilla-Fuentes, L. (2019). La reparación integral de las víctimas con enfoque en la violencia de género. [Comprehensive reparations for victims with a focus on comprehensive gender-based violence]. Revista De Derecho, (25), 04-21. https://doi.org/10.5377/derecho.v0i25.7419

CPD, Red RAP VIF SUR (2017). Ruta de Atención, Protección y Restitución de Derechos de Mujeres en Situación de Violencia en el Marco del Sistema de Protección Integral del DMQ. [Route of Attention, Protection and Restitution of Rights of Women in Situation of Violence within the Framework of the Integral Protection System of the DMQ]. Recuperado de https://url2.cl/7NPPb

Departamento de Derecho Internacional OEA (1994). Convencion Interamericana sobre desaparicion forzada de personas. Adoptada en Belém do Pará, Brasil el 9 de junio de 1994, en el vigésimo cuarto período ordinario de sesiones de la Asamblea General. [Inter-American Convention on the forced disappearance of people. Adopted in Belém do Pará, Brazil on June 9, 1994, at the twenty-fourth regular session of the General Assembly]. Recuperado de https://url2.cl/MajvT

El Telegrafo (2019). Femicidios evidencian pendientes en Derechos Humanos. [Femicides show pending Human Rights]. Recuperado de https://url2.cl/mw766

Gómez, C. (2014). Factores asociados a la violencia: revisión y posibilidades de abordaje. [Factors associated with violence: review and possibilities of approach]. Revista Iberoamericana de Psicología: Ciencia y Tecnología, 7(1), 115-124.

González-Cárdenas, F., Narváez-Zurita, C., Guerra-Coronel, M., \& Erazo-Álvarez, J. (2020). Protección para niños, niñas y adolescentes: La protección integral prevista en la constitución ecuatoriana. [Protection for children and adolescents: The comprehensive protection provided for in the Ecuadorian constitution]. IUSTITIA SOCIALIS, 5(1), 397-414. http://dx.doi.org/10.35381/racji.v5i1.619

Guarderas-Albuja, P. (2016). Silencios y acentos en la construcción de la violencia de género como un problema social en Quito. [Silences and accents in the construction of gender violence as a social problem in Quito]. Revista Íconos, № 55. 191-213. https://doi.org/10.17141/iconos.55.2016.1700

Guzmán, E., Vaca, J., Goyas, L., \& Machado, L. (2019). Aprobación y aplicación de la Ley Orgánica Integral para Prevenir y Erradicar la Violencia contra las Mujeres. [Approval and application of the Comprehensive Organic Law to Prevent and Eradicate Violence against Women]. Revista Metropolitana de Ciencias Aplicadas, 2(2), 44-52. Recuperado de https://cutt.ly/EyloULX 
Hernández, R., \& Mendoza, C. (2018). Metodología de la investigación. Las rutas cuantitativa, cualitativa y mixta. [Investigation methodology. The quantitative, qualitative and mixed routes].Mexico: Mc Graw Hill Education.

Hernández, R., Fernández, C., \& Baptista, M. (2014). Metodología de la Investigación. [Research Methodology]. Mexico DF: McGraw-Hill / Interamericana Editores, S.A. DE C.V.

López-Zambrano, A. (2018). La acción de protección su eficacia y aplicación en el Ecuador. [Protective action, its effectiveness and application in Ecuador].Dominio de las Ciencias, 4(1), 155-177.

Machado, L., Medina, R., Vivanco, G, Goyas, L., \& Betancourt, E. (2018). Reparación integral en el sistema jurídico ecuatoriano ¿derecho público o privado?. [Reparation in the Ecuadorian legal system; public or private right?]. Espacios, 39(9), 14. Obtenido de https://n9.cl/7f554

Martínez-Leguízamo, M. (2018). La reparación integral de la víctima en el delito de violencia física contra la mujer y la familia y su relación con la violencia de género. [The integral reparation of the victim in the crime of physical violence against women and the family and its relationship with gender violence]. Recuperado de https://n9.cl/5akv

Naciones Unidas (2009). Violencia contra las mujeres. [Violence against women]. Recuperado de https://url2.cl/kcLVF

Ordóñez-Segarra, V., Narváez-Zurita, C., Vázquez-Calle, J., \& Erazo-Álvarez, J. (2020). Análisis de la independencia judicial en la administración de justicia del Ecuador. [Analysis of judicial independence in the administration of justice in Ecuador]. IUSTITIA SOCIALIS, 5(1), 584-603. http://dx.doi.org/10.35381/racji.v5i1.631

Organización Mundial de la Salud (2017). Violencia contra la mujer. [Violence against women]. Recuperado de https://url2.cl/TvvGn

Peñafiel-Bermeo, R., Narváez-Zurita, C., Vázquez-Calle, J., \& Erazo-Álvarez, J. (2020). Perspectiva de género en actuaciones y resoluciones judiciales contra la violencia a la mujer. [Gender perspective in judicial actions and resolutions against violence against women]. IUSTITIA SOCIALIS, 5(1), 561-583. http://dx.doi.org/10.35381/racji.v5i1.630 
Peñafiel-Sacoto, J., Erazo-Álvarez, J., Pozo-Cabrera, E., \& Narváez-Zurita, C. (2020). La fundamentación y la motivación como habilitantes de la prisión preventiva. [The rationale and motivation as enablers of preventive detention].IUSTITIA SOCIALIS, 5(8), 465-492. http://dx.doi.org/10.35381/racii.v5i8.586

Quiñonez, H., Zambrano, M., \& Prado, M. (2017). La violencia de género y el derecho penal. [Gender violence and criminal law]. Dominio de las Ciencias, 447-458. doi:10.23857/dom.cien.pocaip.2017.3.4.oct.447-458

Quiñonez-Francis, H., Zambrano-Guerrero, M., \& Prado Solís, M. (2017). La violencia de género y el derecho penal. [Gender violence and criminal law]. Dominio de las Ciencias, 3(4), 447-458

Saccomano, C. (2017). El feminicidio en América Latina. [Femicide in Latin America]. CIDOB, № 117, pp. 51-78. Recuperado de https://url2.cl/PaAut

Salcedo-López, D. (2013). Género, derechos de las víctimas y justicia transicional: Retos en Colombia. [Gender, victims' rights and transitional justice: challenges in Colombia]. Revista De Paz y Conflictos, 6, 124-151. Recuperado de https://url2.cl/Zq7e8

Sánchez, C., \& Oliveros, S. (2014). La reparación integral a las víctimas mujeres: una aproximación a la aplicación del enfoque diferencial de género en el contexto del conflicto armado colombiano. [Comprehensive reparation to women victims: an approach to the application]. Universitas Estudiantes, $\mathrm{N}^{\circ} 11,163-185$. Recuperado de https://url2.cl/c1KA4

Solís, M. (2018). Reparación a víctimas de violación de derechos humanos y crímenes de lesa humanidad en Ecuador. [Reparation to victims of human rights violations and crimes against humanity in Ecuador]. Íconos, 62, 183-201. https://doi.org/10.17141/iconos.62.2018.2826

Taus, P. (2014). La igualdad de género y el acceso a la justicia de las mujeres víctimas de violencia en la región dentro del sistema interamericano de protección de los derechos humanos. [Gender equality and access to justice for women who are victims of violence in the region, within the Inter-American system for the protection of human rights]. Revista IUS, 8(34), 21-41. Recuperado de https://url2.cl/AQiGe

Ubiría, F. (2011). Cambios de paradigma en el derecho de daños. Hacia un nuevo salto de calidad. [Paradigm changes in tort law. Towards a new leap in quality]. Buenos Aires: El Derecho. 
Vásquez-Ayerve, J., Narváez-Zurita, C., Pozo-Cabrera, E., \& Erazo-Álvarez, J. (2020). El alcance de la responsabilidad extracontractual del Estado por sentencia condenatoria reformada 0 revocada. [The scope of the non-contractual responsibility of the State for a conviction amended or revoked]. IUSTITIA SOCIALIS, 5(1), 434-453. http://dx.doi.org/10.35381/racji.v5i1.621

Vélez-Valencia, C. (2017). Abordar la violencia contra las mujeres desde una perspectiva integral. [Addressing violence against women from a comprehensive perspective]. Revista Ciencias de la Salud, 15(2), 299. http://dx.doi.org/10.12804/revistas.urosario.edu.co/revsalud/a.5751

Yugueros-García, A. J. (2014). La violencia contra las mujeres: conceptos y causas. [Violence against women: concepts and causes]. Barataria. Revista CastellanoManchega De Ciencias Sociales, (18), 147-159. https://doi.org/10.20932/barataria.v0i18.49 